\title{
Abschlussprüfung in Deutschland und Europa nach der europäischen Reform von 2014
}

\author{
KLAUS J. HOPT*
}

Inhaltsübersicht

ZGR 2015, 186-203

I. Die Abschlussprüferreform von 2014 . . . . . . . . . . . . . . . . . . . . 187

1. Die europäische Reform des Abschlussprüferrechts vom 16. April/27. Mai

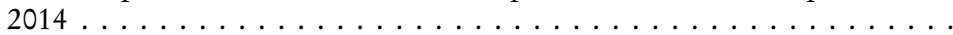

2. Kernpunkte der Abschlussprüferverordnung nach den Vorstellungen der Europäischen Kommission und der endgültigen Verabschiedung . . . . . .

3. Erwartungen zur externen Rotation im Besonderen . . . . . . . . . . .

4. Bemerkungen zu den nicht realisierten Reformvorschlägen der Europäischen Kommission. . . . . . . . . . . . . . . . . . . .

5. Zuständigkeitsfragen: Nationale Aufsichtsbehörden und ESMA . . . . . .

II. Harmonisierung eines Kernbereichs der Abschlussprüferhaftung zwecks gröBerer Rechtssicherheit . . . . . . . . . . . . . . .

III. Internationalisierung des Wirtschaftsprüferrechts . . . . . . . . . . . . . 199

IV. Abschlussprüfer zwischen Vorstand und Aufsichtsrat . . . . . . . . .

Der Beitrag behandelt die Reform des europäischen Abschlussprüferrechts (Verordnung und Richtlinie vom 16.4.2014), die in Deutschland sebr kontrovers diskutiert worden ist. Kernpunkte sind schärfere Bestimmungen über die Unabhängigkeit und Vermeidung von Interessenkonflikten, erböbte Anforderungen an die Durchfübrung der Abschlussprüfung und vor allem eine externe Rotation. Kurz eingegangen wird auch auf die nicht realisierten Reformvorschläge der Europäischen Kommission sowie Zuständigkeitsfragen (nationale Aufsichtsbehörden und ESMA). Weitere in dem Aufsatz behandelte Themen sind die Harmonisierung des Abschlussprïferrechts, seine Internationalisierung (ISA) sowie die Stellung des Abschlussprüfers zwischen Vorstand und Aufsichtsrat, also corporate governance und Abschlussprüfer.

This article deals with the European auditing law reform (Regulation and Directive of 16 April 2014). The discussion in Germany was very controversial. Main points of the reform are stricter rules on the independence and avoidance of conflicts of interest, higher requirements as to auditing and in particular the external rotation of audit firms. The reform agenda that has not been enacted will also be treated briefly as well as questions of competence of the national supervisory bodies and the ESMA. Further topics dealt with are harmonization of

* Der Autor ist Professor und Direktor em. am Max-Planck-Institut für ausländisches und internationales Privatrecht in Hamburg und Mitherausgeber der ZGR. Der Beitrag stellt die erweiterte, auf die europäische Abschlussprüferrechtsreform hin à jour gebrachte Fassung einer Einführung in das Sondersymposium der ZGR über „Die Zukunft der Abschlussprüfung in Deutschland und Europa" am 23.11.2013 in Frankfurt dar. 
auditing law, its internationalization (International Standards on Auditing) as well as the role of the auditors between the management board and the supervisory board, i.e. corporate governance.

Das Sondersymposium der ZGR über „Die Zukunft der Abschlussprüfung in Deutschland und Europa" am 23.11.2013 in Frankfurt hat einen kleinen, ausgewählten Kreis zusammengebracht, um die schwierigen und zum Teil hoch kontroversen Fragen der „Zukunft der Abschlussprüfung in Deutschland und Europa“ zu diskutieren, vielleicht zu klären und möglicherweise sogar den einen oder anderen rechtspolitischen Vorschlag zu machen. In der Zwischenzeit sind die europäischen Reforminstrumente am 16.4.2014 verabschiedet und am 27. 5.2014 veröffentlicht worden. Es handelt sich um die Verordnung (EU) Nr. 537/2014 zur Abschlussprüfung bei Unternehmen von öffentlichem Interesse ${ }^{1}$ und die Richtlinie 2014/56/EU zur Änderung der Richtlinie 2006/43/EG über Abschlussprüfungen von Jahresabschlüssen und konsolidierten Abschlüssen. ${ }^{2}$ Die Abschlussprüferverordnung enthält unmittelbar verbindliches Europarecht und gilt ab 17.6.2016 mit Ausnahme von Art. 16 Abs. 6 betreffend das Verbot von Vertragsklauseln zur Auswahl des Abschlussprüfers, das erst ab 17.6. $2017 \mathrm{zu}$ beachten ist. Die Abschlussprüferrichtlinie in der Fassung der Änderungsrichtlinie muss von den Mitgliedstaaten bis zum 17.6. 2016 in nationales Recht umgesetzt werden. Die ZGR gibt im Folgenden sechs Beiträge zu dem Sondersymposium in einer aktualisierten Fassung wider, zwei zu Problemen der Europäischen Rechtssetzung im Abschlussprüferrecht, einen zur Frage der Haftung des gesetzlichen Abschlussprüfers und drei zur Internationalisierung des Wirtschaftsprüferrechts. Die Beiträge stehen exemplarisch für den Dialog zwischen der Rechtswissenschaft (W. Doralt, Hennrichs, und Merkt), den Wirtschaftswissenschaften (Böcking und Köbler) und der Praxis (Poll). Einführend waren zu den Themenkomplexen der Veranstaltung - europäische Rechtssetzung, Internationalisierung und Abschlussprüfer zwischen Aufsichtsrat und Vorstand - einige kurze, einführende Überlegungen beigesteuert worden. Diese werden im Folgenden in aktualisierter Fassung wiedergegeben.

\section{Die Abschlussprüferreform von 2014}

\section{Die europäische Reform des Abschlussprüferrechts vom 16. April/27. Mai 2014}

Akuter Anlass der Themenwahl für das Sondersymposium waren die insgesamt zu ambitionierten Reformvorschläge der Europäischen Kommission vom 30.11.2011, die bekanntlich eine erbitterte, berufliche, wissenschaftliche

2 Sog. Abschlussprüfungsänderungsrichtlinie, ABlEU L 158/196 ff. 
und politische Diskussion hervorgerufen haben. ${ }^{3}$ Sie gehen zurück oder sind jedenfalls maßgeblich geprägt von der Finanzkrise und der Vorstellung, die Wirtschaftsprüfer hätten dabei versagt. ${ }^{4}$ Auch die Umfrage der Kommission zu ihrem Grünbuch hat eine Rolle gespielt. ${ }^{5}$ Die Pläne der Kommission, die Abschlussprüfungsrichtlinie ${ }^{6} \mathrm{zu}$ erweitern und sie um eine Abschlussprüferverordnung zu ergänzen, ${ }^{7}$ sind mittlerweile realisiert, wenn auch nicht so, wie die Kommission sich das vorgestellt hat. Die Reform der Abschlussprüfungsrichtlinie beinhaltet unter anderem einen weiteren Begriff der Abschlussprüfung, einen europäischen Pass, das Bestehen auf einer „kritischen Grundhaltung “, Vorgaben zur internen Organisation und Arbeitsorganisation, Prüfung und Bestätigungsvermerk gemäß den internationalen Prüfungsstandards und Erleichterungen für kleine und mittlere Unternehmen, sofern diese nicht Unternehmen von öffentlichem Interesse sind. So weit so gut. Die eigentlichen Probleme liegen jedoch in der Abschlussprüferverordnung. Hier wurden die Anforderungen an die Abschlussprüfung bei Unternehmen von öffentlichem Interesse (public interest companies, PIE) ${ }^{8}$ ganz erheblich verschärft. Vor allem aber sind sie mit dem Übergang auf die Rechtsform der europäischen Verordnung unmittelbar unionsrechtlich geregelt. Das ist eine in den letzten Jahren besonders im Bank- und Kapitalmarktrecht aufgekommene Praxis, zu der vorhergesagt wird, dass künftig „ein Großteil des Kapitalmarktrechts“ als Verordnung erlassen werden wird. ${ }^{9}$ Diese Praxis sorgt zwar für größere Rechtseinheitlichkeit, aber um den Preis, dass sie den Mitgliedstaaten die Möglichkeit systemkonformer Umsetzung in das nationale Recht entzieht

3 Z. B. Schwerpunkt: Regulierung, Audit Committee Quarterly II/2014, 4 ff; kurzer Überblick über die $\$ \$ 316-324$ a HGB im europäischen und internationalen Umfeld bei Норт/Merkт, in: Baumbach/Hopt, HGB, 36. Aufl., 2013, Überblick vor $\$ 316$ Rdn. 3 ff.

4 Prototypisch für die USA der einflussreiche Gesellschaftsrechtler CoffeE von der Columbia University mit seinem Buch: Gatekeepers: The Role of the Professions and Corporate Governance, 2006. Die Ursachen der Finanzkrise sind allerdings komplex und liegen nicht primär an Corporate Governance-Mängeln bei den Wirtschaftsprüfern oder bei den Finanzinstituten, vgl. Норт, Corporate Governance of Banks and Other Financial Institutions After the Financial Crisis, Journal of Corporate Law Studies 2013, 219 at 237 et seq.

5 Kritisch dazu Böcking/Gros/Wallek/Worret, Analysis of the EU Consultation on the Green Paper „Audit Policy: Lessons from the Crisis“, Studien des Deutschen Aktieninstituts, 2011, Heft 51.

6 Europäische Kommission, Vorschlag für eine Richtlinie zur Änderung der Richtlinie 2006/43/EG über Abschlussprüfungen von Jahresabschlüssen und konsolidierten Abschlüssen vom 30.11.2011, KOM(2011) 778 endgültig.

7 Europäische Kommission, Vorschlag für eine Verordnung über spezifische Anforderungen an die Abschlussprüfung bei Unternehmen von öffentlichem Interesse vom 30.11.2011, $\operatorname{KOM}(2011) 779$ endgültig.

8 Kapitalmarktorientierte Unternehmen, Kreditinstitute, Versicherungen.

9 VEIL, Europäisches Kapitalmarktrecht, 2. Aufl., 2014, \$3 Rdn. 14 mit Beispielen (Ratingagenturen 2009, Leerverkäufe 2012, Insiderverbote und Marktmanipulationen 2014). 
und die unvermeidlichen Auslegungsprobleme direkt auf die europäische Ebene hinaufschiebt. Der Rechtsausschuss des Europäischen Parlaments hatte deswegen in seinem Bericht vom 15. April 2013 vorgesehen, einen Teil der Artikel aus der Verordnung in die Richtlinie zu überführen. Die nunmehr zustande gekommene Abschlussprüferreform war bis zuletzt strittig und wird dementsprechend diametral unterschiedlich bewertet. ${ }^{10}$ Für Deutschland kommt es jetzt auf die Umsetzung durch den Gesetzgeber an, vor allem was die Mitgliedsstaatenwahlrechte angeht und ob das deutsche berufsständische System, was die Aufsicht angeht, unverändert beibehalten werden kann. ${ }^{11}$

\section{Kernpunkte der Abschlussprüferverordnung nach den Vorstellungen der Europäischen Kommission und der endgültigen Verabschiedung}

Schon in dieser Einführung sollten drei Knackpunkte der Verordnung benannt werden, die nur Unternehmen von öffentlichem Interesse betreffen (die von der Kommission vorgesehene wesentliche Ausdehnung dieses Begriffs hat der Rechtsausschuss abgelehnt). ${ }^{12}$ Sie betreffen:

a) Schärfere Bestimmungen über Unabhängigkeit und Vermeidung von Interessenkonflikten. Was bedeutet das konkret? Nach den Vorstellungen der Kommission: eine sehr weitgehende Regelung zu Verbot bzw. Untersagung bestimmter prüfungsfremder Leistungen; Höchstgrenze von 10\% des Prüfungshonorars von dem Unternehmen für prüfungsverwandte Leistungen für das Unternehmen; Bestätigung der Unabhängigkeit durch den Prüfungsausschuss; Anforderungen an die interne Organisation und Karenzzeit bei Einstellung von früheren Abschlussprüfern oder Mitarbeitern von zwei Jahren.

In der endgültigen Verordnung wurde für den Hauptstreitpunkt des Katalogs verbotener Nichtprüfungsleistungen folgender politischer, nicht unbedingt ökonomisch ${ }^{13}$ begründbarer Kompromiss erzielt (Art. 5). Ausgeschlossen sind

10 Vgl. einerseits Hennrichs und Böcking/Gros, in diesem Heft, S. 265 unter III. bzw. S. 318 unter V.: weitgehend nicht sachgerecht und nicht zielführend; andererseits z. B. Fockenbrock, Zahmer Bettvorleger, Handelsblatt 19.12. 2013, Nr. 245 S. 27.

11 Hennrichs, in diesem Heft, S. $258 \mathrm{ff}$ unter II. 2. b) dd). Dazu zuletzt RefE BMJV 27.3. 2015 und demnächst Merkt ZHR 179 (2015).

12 Einen ausführlichen Überblick über die Reform betreffend die Abschlussprüfung bietet Scheffler, AG 13-14/2014 R 196 ff, betreffend den Prüfungsausschuss Scheffler, AG 20/2014 R 304; zuvor Naumann/Herkerdell, WPg 2014, 177. Zu den Vorschlägen der Kommission kritisch Arbeitskreis Bilanzrecht Hochschullehrer RechtswisSENSchaft, NZG 2012, 294; Velte, EWS 2012, 220; auch kurz Verse, EuZW 2013, 336, $339 \mathrm{f}$ und Verse/Wiersch, EuZW 2014, 375, 379.

13 Dazu Böcking/Gros, in diesem Heft, unten S. 309 ff unter III. 1. mit Tabellen 1 und 2 über die einschlägigen ökonomischen Studien. Auch KöHLER/Lıu, WPg 2014, 985 mit einem Überblick über die jüngste internationale Prüfungsforschung; BöcKING/Gros/ 
nunmehr unter anderem Steuerberatungsleistungen, Leistungen im Bereich des Rechnungswesens und der internen Revision, Bewertungsleistungen, juristische Beratung und Unterstützung, Leistungen bei Finanzierung, Kapitalausstattung und Anlagestrategie sowie Personaldienstleistungen bezüglich der Mitglieder der Unternehmensleitung, des Aufbaus der Organisationsstruktur und der Kostenkontrolle. Unter bestimmten Voraussetzungen sind Ausnahmen möglich, allerdings keinesfalls bei Teilnahme an den Entscheidungsprozessen, Erbringung von Leistungen auf dem Gebiet des Rechnungswesens und Gestaltung und Umsetzung interner Kontroll- und Risikomanagementverfahren. ${ }^{14}$

b) Erhöbte Anforderungen an die Durchfübrung der Abschlussprüfung, also an Organisation und Ressourcen und an Qualitätssicherung - dies alles mit einer, wie es heißt, „kritischen Grundhaltung“ (professional scepticism); ${ }^{15}$ Bestätigungsvermerk und zusätzlicher Bericht zur Abschlussprüfung an den Prüfungsausschuss, nunmehr Art. 11 der Verordnung; weiter erhöhte Transparenz, insbesondere ein Transparenzbericht (Art. 13) und Meldungen an die zuständige Behörde bei wesentlichen Verstößen und ähnlichen Fällen $\left(\right.$ Art. 12) ${ }^{16}$; Empfehlung des Prüfungsausschusses zur Bestellung des Abschlussprüfer.

c) Vor allem aber eine externe Rotation. Diese stellte sich die Kommission so vor: Erstbestellung für mindestens zwei Jahre, Wiederbestellung nur bis zu insgesamt sechs Jahren, ausnahmsweise Verlängerung auf acht Jahre; bei Befassung von zwei Abschlussprüfern neun Jahre, ausnahmsweise bis zu zwölf Jahren; dann Karenzzeit von vier Jahren, Übergabebericht an den neuen.

Die verabschiedete Fassung hat die externe Rotation zwar wie vorhergesagt beibehalten, aber deutlich abgemildert: Art. 17 der Verordnung schreibt einen Prüferwechsel grundsätzlich nach 10 Jahren vor. Nach Ablauf dieser Höchstlaufzeit müssen Unternehmen von öffentlichem Interesse den Abschlussprüfer bzw. die Abschlussprüfergesellschaft völlig wechseln, die externe Rotation ist also insoweit geltendes Europarecht. Die Verordnung unterscheidet zwischen einem ersten und einem verlängerten Mandat. Das erste Mandat muss mindestens ein Jahr betragen. Es kann verlängert werden. Insgesamt darf jedoch die Höchstlaufzeit des ersten und, falls es dazu kommt, des zweiten,

Worret, Regulierung der Abschlussprüfung - eine Analyse der aktuellen Entwicklungen in Theorie und Praxis, Jahrbuch für Controlling und Rechnungswesen 2013, S. 449.

14 Genauer Art. 5 UAbs. 3 lit. a und b der VO (Fn. 1), ABIEU L 158/88; LANFermann, BB 2014, 1771 (Nichtprüfungsleistung).

15 Dazu Koch/Worret, Der Konzern 2013, 475; Chekushina/Loth, NZG 2014, 85; Deckers/Hermann, DB 2013, 2315.

$16 \mathrm{Zu}$ Letzterem distanziert Arbeitskreis Bilanzrecht Hochschullehrer RechtsWISSENSCHAFT, NZG 2012, 294, 295/296. 
verlängerten Mandats die 10 Jahresfrist nicht überschreiten. Mitgliedstaatenoptionen bestehen insoweit, als das erste Mandat länger dauern kann und die Höchstlaufzeit verkürzt werden darf.

Die Regelhöchstlaufzeit von 10 Jahren kann - so der schließlich zustande gekommene Kompromiss - ausnahmsweise auf 20 und sogar auf 24 Jahre verlängert werden. Ersteres setzt die Durchführung eines öffentlichen Ausschreibungsverfahrens voraus, letzteres die Beauftragung von mehr als einem Abschlussprüfer mit der Vorlage eines gemeinsamen Bestätigungsvermerks. Für Verlängerungen werden vorausgesetzt eine Empfehlung durch den Prüfungsausschuss des Aufsichtsrats, ein entsprechender Vorschlag des Aufsichtsrat und die Annahme dieses Vorschlags durch die Hauptversammlung. Ganz ausnahmsweise kommt noch eine Verlängerung um bis zu zwei Jahre durch die Aufsichtsbehörde in Betracht. Sehr großzügige Übergangsfristen ${ }^{17}$ mildern die externe Rotation weiter ab. Hinzu kommt eine interne Rotation der verantwortlichen Prüfungspartner nach spätestens sieben Jahren für mindestens drei Jahre, während für das an der Abschlussprüfung beteiligte Führungspersonal ein „angemessenes graduelles Rotationssystem“ einzuführen ist. ${ }^{18}$

\section{Erwartungen zur externen Rotation im Besonderen}

Über die Zukunft der externen Rotation, die nunmehr Gesetz geworden, gibt es unterschiedliche Voraussagen. Die empirischen Befunde und den letzten Stand der Diskussion aus betriebswirtschaftlicher Sicht stellen Böcking und Gros vor. ${ }^{19} \$ 319$ a Abs. 1 Nr. 4 HGB sieht bekanntlich nur einen personellen Prüferwechsel vor. Den zu weit gehenden Vorschlag der Europäischen Kommission zur zwingenden externen Rotation hatte schon der Rechtsausschuss des Europäischen Parlaments ganz erheblich zurückgestutzt. Vorgesehen war derzeit eine maximale Mandatsdauer von 14 Jahren, die unter bestimmen Umständen (Ausschreibung, Gemeinschaftsprüfung oder audit engagement) bis zu 25 Jahren verlängert werden konnte. Dabei ist es mit geringfügigen Änderungen in der europäischen Gesetzgebung am Ende trotz aller Argumente der Betriebswirtschaft und des Berufsstands ${ }^{20}$, wie auch in dieser Einführung zu dem Symposium vorhergesagt, geblieben. In der Diskussion war

17 Art. 41 der VO (Fn. 1), ABlEU L 158/111 f. Dazu Hennrichs, in diesem Heft, S. 258 ff unter II. 2. b) dd). Voraussichtlich werden nicht alle Unternehmen diese Fristen ausschöpfen, um nicht zu zu vielen Wechseln im Jahre 2020 und später zu kommen.

18 Art. 17 Abs. 7 U Abs. 1 und 3 der VO (Fn. 1), ABlEU L 158/98.

19 BöcKIng/Gros, in diesem Heft, S. 307 ff.

20 Zuletzt Köhler/Gehring, BB 2015, 235. Aber demgegenüber P. Doralt, Die Abhängigkeit des Abschlussprüfers: Gedanken zur externen Rotation, in: Allmendinger/ Dorn/Lang/Lumpp/Steffek, Corporate Governance nach der Finanz- und Wirtschaftskrise, 2011, S. 179. 
zwar noch die Option, (wirksame) Alternativen zur externen Rotation im Hinblick auf die Sicherung der Unabhängigkeit der Abschlussprüfer zu wählen. Doch ist das nur ansatzweise im Rahmen der Ausnahme von der Regellaufzeit von zehn Jahren Gesetz geworden. Eine Fundamentalopposition, wie sie in Deutschland lange geübt worden war, erscheint unter diesen Umständen wenig sinnvoll. Überlegt und vertieft werden könnte stattdessen Folgendes.

Zum einen gibt es in der Europäischen Union die externe Rotation nur in Italien und die Befunde der vorliegenden empirischen Untersuchungen zu den Wirkungen der externe Rotation sind uneinheitlich, ${ }^{21}$ sodass sich daraus keine belastbaren Schlüsse auf eine positive Wirkung der Einführung einer externen Rotation ergeben. Die daraus gezogene Folgerung, diese hätte dann ganz unterbleiben müssen, greift aber zu kurz. Der Gesetzgeber, der unter dem Druck der Erwartungslücke steht, muss dann eben wie häufig unter Unsicherheit entscheiden und tut das auch, und stützt sich dann in einem solchen Fall wie hier auf allgemeine Argumente zur Stärkung des Vertrauens des Anlagepublikums auf die Abschlussprüfer und ihre Funktion im öffentlichen Interesse. ${ }^{22}$ Subsidiaritäts- und verfassungsrechtliche Einwände, wie gerade in Deutschland verbreitet, haben dagegen wenig Aussicht auf Erfolg. ${ }^{23}$ Vielmehr käme es, nachdem der europäische Gesetzgeber sich nun einmal für die externe Rotation entschieden hat, darauf an, empirisch umgekehrt nachzuweisen, dass die externe Rotation schädliche Wirkungen hat. Das könnte sich möglicherweise im Hinblick auf die Folgen für die Konzentration am Markt darstellen lassen, etwa wenn die externe Rotation nur dazu führen würde, dass ein „Bäumchen wechsle dich“ unter den big four einträte. Die Frage wäre dabei, ob etwa für den Bankbereich nicht auch die Folge sein könnte, dass neue Wettbewerber Know-how aufbauen oder einzelne Teams von den zwei dominierenden Wirtschaftsprüfungsgesellschaften zu einer anderen überwechseln und auf diese Weise einen neuen Konkurrenten schaffen würden. Die Voraussagen dazu sind diametral unterschiedlich: Die einen erhoffen sich eine Auflockerung des Vierer-Oligopols hin auf das darunter liegende Mittelfeld von sechs oder sieben oder sogar 10 bis 15 Prüfungsgesellschaften, die anderen befürchten, dass die Reform die Konzentration eher noch weiter fördert. ${ }^{24}$ Was davon tatsächlich eintreten wird, wird sich erst nach Jahren herausstellen.

21 Vgl. z. B. Sipple/Glemser, WPg 2012, 875; Ewelt-Knauer/Gold/Pott, WPg 2013, 125; Quick, ZCG 2013, 73. Ebenso Böcking/Gros, in diesem Heft, S. 313 unter III. 2.: keine einheitlichen Forschungsergebnisse.

22 Präambel 1 der VO (Fn. 1), ABlEU L 158/77.

$23 \mathrm{Zu}$ diesen Hennrichs, in diesem Heft, S. $253 \mathrm{ff}$ unter II. 2. b) bb), cc).

24 Naumann (IDW): Qualitätsverlust und weitere Konzentration, Handelsblatt 18. 12. 2013, Nr.244, S. 19. Aus der Wirtschaftspresse z. B. Giersberg, FAZ 1.7.2014, Nr. 149, S. 24; Branche der Wirtschaftsprüfer ist zerstritten, FAZ 6. 1.2014, Nr. 4 S. 24. 
Zum anderen müsste man sich noch einmal mit dem ganz überwiegend vorgebrachten und auch gemeinhin akzeptierten Grund auseinandersetzen, dass bei externer Rotation wegen der Einarbeitungszeit für die neue Prüfungsgesellschaft Qualitätsverluste der Prüfung in den ersten Jahren nach dem Wechsel zu befürchten sind, wie das ausdrücklich auch der Regierungsentwurf des WPOÄG 2000 erwähnt. Auch insoweit sind die internationalen empirischen Befunde uneinheitlich und differenziert. Richtig ist sicher, dass die Zeit nach dem Wechsel problematisch ist. Problematisch ist es aber unter dem Gesichtspunkt der Corporate Governance und der unabhängigen Kontrolle auch, wenn eine Gesellschaft wie zahlreiche DAX-Unternehmen seit zwanzig oder mehr Jahren - als Extrembeispiel wird aus England für Barclays die Zahl von 117 Jahren ohne Wechsel berichtet - denselben Abschlussprüfer hat. Auf der anderen Seite hat eine Abschlussprüfungsgesellschaft, die jedes Jahr neu bestellt werden muss, rechtlich (nicht tatsächlich) keine Planungssicherheit über mehrere Jahre. Die empirische Frage ist, wie nachteilig das tatsächlich ist und ob eine Reformkombination von längerem Bestellungszeitraum und im Gegenzug externer Rotation nicht sinnvoller wäre. ${ }^{25}$

Wegen dieser Übergangs- und Einarbeitungsschwierigkeiten muss drittens eine externe Rotation unbedingt mit einer Rechtsnorm gekoppelt werden, die sicherstellt, dass die neue Gesellschaft von der alten nicht nur, wie bei Kündigung des Prüfungsauftrags nach $\$ 318$ Abs. 5 und 6 HGB, den Prüfungsbericht an die Gesellschaft erhält und der Mandatsvorgänger berufsrechtlich verpflichtet ist, diesen zu erläutern, ${ }^{26}$ sondern dass er von diesem ungeachtet der normalen Verschwiegenheitspflicht des (vorigen) Prüfers vollen Einblick in die Fragen und Probleme der geprüften Gesellschaft erhält. Das ist nämlich das eigentliche Problem und der Kommissionsvorschlag einer Verordnung enthält dazu in Art. 33 Absatz 5 auch ansatzweise eine Regelung (insbesondere Übergabebericht und Zugang zu den Berichten an den Prüfungsausschuss). ${ }^{27}$

Vgl. auch Backhaus/Kirsch/Kraft, Perspektiven des Berufsstandes der Wirtschaftsprüfer 2025, IDW-FN Beiheft zu Nr. 2/2015; АкEIÜ, DB 2014, 1149.

25 Eine Arbeitsgruppe des Hamburger Max-Planck-Instituts hat als quid pro quo vorgeschlagen, einerseits einen Bestellungszeitraum auf vier Jahre mit einer einmaligen Verlängerungsmöglichkeit vorzusehen, andererseits dann aber eine externe Rotation zu verlangen. W. Doralt/Hellgardt/Hopt/Leyens/M. Roth/Zimmermann, Auditors' Liability and its Impact on the European Financial Markets, Cambridge Law Journal 67(1) March 2008, 62. So auch schon P. Doralt, aaO (Fn. 20), S. 196, 203. Dazu soeben auch Arbeitskreis Bilanzrecht Hochschullehrer Rechtswissenschaft, Für eine mehrjährige Bestellperiode des Abschlussprüfers, BB 2015, 555, und Hommelhoff/Lanfermann, FS Haarmann, 2015 (im Druck).

26 Eвке, in: Münchener Komm. z. HGB, 3. Aufl., 2013, \318 Rdn. 93 und 94.

$27 \mathrm{Ob}$ diese so ausreicht, ist fraglich. Der neue Abschlussprüfer müsste vom alten regelrecht eingeführt werden und ein Auskunftsrecht gegenüber diesem haben. Fraglich könnte u. a. sein, ob Art. 33 Abs. 5 Unterabs. 1 Satz 2 über Zweck und Inhalt des Übergabeberichts Schutzwirkung entfaltet. 
In der Abschlussprüfungsverordnung ist das dahin modifiziert worden, dass der frühere Prüfer dem neuen Prüfer Zugang zu dem neu eingeführten „zusätzlichen Bericht an den Prüfungsausschuss “ ${ }^{28}$ nach Art. 11 hinsichtlich früherer Jahre ${ }^{29}$ sowie zu jeglichen Informationen an die zuständigen Behörden nach Art. 12 und 13 (Transparenzbericht) gibt, die sogenannte Übergabeakte nach Art.18. Diese Berichte und Informationen sind in der Verordnung so weit und detailliert gefasst, dass der neue Prüfer einen guten Einblick in die Aufgabe bekommt, die er übernimmt. Ob das in der Praxis so funktionieren wird, dass damit die Problematik der Einarbeitungsschwierigkeiten bewältigt oder jedenfalls hinreichend entschärft wird, wird die Zukunft zeigen. Zu bedenken gewesen wären aber auch eine ganze Reihe anderer und weiterer Probleme und Lösungsvorschläge wie etwa die in der Diskussion befindliche Verschärfung für Finanzinstitute oder die Berücksichtigung der Belange internationaler Konzerne ${ }^{30}$ in solchen Fällen.

Bei alledem und vor allem darf nicht vergessen werden, dass Unabhängigkeit wichtig ist, aber nicht zum alleinigen Ziel werden darf. Wie aus der Corporate Governance-Diskussion bekannt, ist der frühere Glaube in die allein seligmachende Funktion von independent directors seit der Finanzkrise der Erkenntnis gewichen, dass vor allem auch die Erfahrung und Kompetenz wichtig sind. ${ }^{31}$

\section{Bemerkungen zu den nicht realisierten Reformvorschlägen der Europäischen Kommission}

Der Widerstand der Mitgliedstaaten gegen die weitgehenden Pläne der Barnier-Kommission war heftig. ${ }^{32}$ Dabei sind auch die von der Europäischen Kommission formulierten Befragungsergebnisse des Konsultationsprozesses

28 Einen solchen hält unabhängig von der externen Rotation auch für Non-PIEs für sinnvoll Arbeitskreis Bilanzrecht Hochschullehrer Rechtswissenschaft, NZG 2012, 294, 295.

29 Wie viele das sein müssen, wird hier nicht gesagt. Auch sonst gibt es in der Verordnung und in der Richtlinie zahlreiche Auslegungsspielräume.

30 Zum Letzteren P. Doralt, aaO (Fn. 20), S. 196.

$31 \mathrm{Zu}$ den diesbezüglichen Befunden der vergleichenden Corporate Governance Hopt, ZHR 175 (2011), 444, 472 ff, 475.

32 Dazu schon zuvor Arbeitskreis Bilanzrecht der Hochschullehrer RechtswisSENSCHAFT, Stellungnahme vom 8.10.2010 zum Grünbuch der Europäischen Kommission, Weiteres Vorgehen im Bereich der Abschlussprüfung: Lehren aus der Krise; MaXPlanck-Institut-Arbeitsgruppe zur Unabhängigkeit der Abschlussprüfer, Stellungnahme zum Grünbuch der Europäischen Kommission „Weiteres Vorgehen im Bereich der Abschlussprüfung: Lehren aus der Krise“, ZIP 2011, 459; auch in: Comments on the European Commission Green Paper, Rivista delle Società 56 (2011) 485; überarbeitete Fassung in: Auditor Independence at the Crossroads - Regulation and Incentives, European Business Organization Law Review (EBOR) 13 (2012) 89. 
kritisch hinterfragt worden. ${ }^{33}$ Besonders strittig waren die externe Rotation und die Inkompatibilitäten und hier das Verbot der Simultanberatung und das Gebot reiner Prüfungsgesellschaften mit Prüfungseinnahmen von mehr als 1,5 Mrd. Euro (pure audit firms). Ganz erhebliche Änderungen an den beiden Vorschlägen hatte sich schon im Rechtsausschuss abgezeichnet, ${ }^{34}$ später bei der Festlegung der Verhandlungsposition des Ministerrats unter Leitung der Ratspräsidentschaft und dann im Trilogverfahren. Das wird aus juristischer Sicht von Hennrichs und aus ökonomischer Sicht von Böcking und Gros näher behandelt. ${ }^{35}$

Das von Kommissar Michel Barnier aus Frankreich ${ }^{36}$ übernommene Gebot der Einschaltung von zwei Prüfungsgesellschaften ist nur noch als Rudiment in der Präambel und im Zusammenhang mit der externen Rotation übrig geblieben, dort wie bereits erwähnt als eine der Voraussetzungen für eine Verlängerung der Höchstlaufzeit auf 24 Jahre. ${ }^{37}$ Tatsächlich hätte eine solche Doppelbeauftragung keine greifbaren Vorteile und würde nur die Prüfung verteuern, auch wenn eine solche Vorschrift von manchen kleinen und mittleren Prüfungsunternehmen gewiss gern gesehen worden wäre. Schon der Rechtsausschuss hatte das zu Recht gestrichen. Aus rechtsvergleichender Sicht ist auch anzumerken, dass es nicht unproblematisch wäre, wenn ein Kommissar ein Institut, das im wesentlichen nur in seinem eigenen Land bekannt ist, allen übrigen Mitgliedstaaten nicht nur als Option anbieten, sondern durch Richtlinien- oder sogar Verordnungsrecht aufzwingen wollte.

Auch das faktische Beratungsverbot für Abschlussprüfer und die pure audit firms sind gefallen und, wie oben erwähnt, durch moderate Grenzen der Beratungstätigkeit ersetzt worden. Niemandem wäre damit gedient, wenn sich Wirtschaftsprüfungsgesellschaften aus dem Tätigkeitsfeld der Abschlussprüfung aus verständlichen ökonomischen Überlegungen zurückziehen und anderen Geschäftsmodellen zuwenden würden. Das wird derzeit in einem anderen Bereich - der Anlegerkundenberatung durch die Banken - angesichts zu weit gehender Anforderungen durch den Gesetzgeber und die Rechtsprechung mit Sorge be-

33 Böcking/Gros/Wallek/Worret, aaO (Fn. 5).

34 EU-Regulierung der Abschlussprüfung: Zwischenstand nach Abstimmung im Rechtsausschuss, IDW Fachnachrichten Nr. 6/2013, 254.

35 Hennrichs, in diesem Heft, S. 249 ff; Böcking/Gros, in diesem Heft, S. $305 \mathrm{ff}$.

36 Zeitweise auch in Dänemark, außerdem vereinzelt freiwillige joint audits, z. B. bei der Deutschen Telecom. Zu den kaum aussagekräftigen Erfahrungen mit solchen joint audits Böcking/Gros, in diesem Heft, S. $316 \mathrm{f}$ unter III. 3.; RATZINGER-SAKEL/LAMBACHER, DB 2013, 1369. Auch IDW Prüfungsstandard: Zur Durchprüfung von Gemeinschaftsprüfungen (Joint Audit), Stand 24. 11. 2010, IDW PS 208, FN-IDW 2011, 113 f.

37 Die in eine Beauftragung von mehr als einem Abschlussprüfer gesetzten Hoffnungen (Erhöhung der Prüfungsqualität und, ohne dass das so gesagt würde, Auflockerung des Oligopols) finden sich noch in Präambel 20 und 21 der VO (Fn. 1), ABlEU L 158/81. 
obachtet. Unter den Wirtschafts- und Buchprüfern, ist zu hören, sind schon heute nur noch $61 \%$ im Umfeld der Abschlussprüfung tätig.

\section{Zuständigkeitsfragen: Nationale Aufsichtsbehörden und ESMA}

Über die inhaltlichen Fragen, die in der Diskussion verständlicherweise ganz im Vordergrund standen, dürfen die kompetenziellen, Durchsetzungs- und Aufsichtsfragen nicht vergessen werden. Diese sind längerfristig möglicherweise sogar noch relevanter. Die Unternehmen des öffentlichen Interesses werden durch eine oder (nach dem Rechtsausschuss) mehrere zuständige Behörden ${ }^{38}$ beaufsichtigt. Die Kommission hatte vorgesehen, dass die nationalen Behörden nicht nur EU-weit zusammenarbeiten - dagegen gibt es natürlich nichts einzuwenden, im Gegenteil, das ist erwünscht -, sondern dass die Zusammenarbeit im Rahmen der ESMA ${ }^{39}$ in Paris organisiert werden sollte und, vor allem, dass die ESMA dann zu bestimmten Punkten Leitlinien erlassen können sollte. Zur Betrauung der ESMA als Kernkoordinationsstelle (core structure for coodination) ist es aber nicht gekommen, was Kommissar Barnier ausdrücklich bedauert hat. ${ }^{40}$ Vielmehr werden Kollegien der zuständigen nationalen Behörden gebildet und ein Ausschuss der Aufsichtsstellen eingesetzt mit einem Mitglied je Mitgliedstaat und einem von der ESMA benannten Mitglied, Letzteres hat aber kein Stimmrecht. ${ }^{41}$ Der Ausschuss wird aber auf sein Ersuchen hin von der ESMA, EBA und EIOPA unterstützt.

Die Natur dieser von der Kommission für die ESMA vorgesehenen, nunmehr vom Ausschuss der Aufsichtsstellen zu verabschiedenden Leitlinien ist unklar. ${ }^{42}$ Rechtsverordnungen sind sie nicht, bloße Empfehlungen aber, obwohl sie ausdrücklich als unverbindlich bezeichnet werden, auch nicht, zumal die Kommission sie veröffentlicht. ${ }^{43}$ Erwartet wird vielmehr die Befolgung solcher Leitlinien, denn bei Nichtbefolgung ist nicht wie beim Deutschen Corporate Governance Kodex nach $\mathbb{} 161$ AktG einfach über Offenlegung ein

38 Was das für die deutsche berufsständische Aufsicht bedeutet, ist bislang offen und hängt für das Erste von der deutschen Umsetzung ab.

$39 \mathrm{Zu}$ dieser und ihrer Rolle im der europäischen Kapitalmarktaufsicht ausführlich VeIL, aaO (Fn. 9), $\mathbb{1} 11$ Rdn. $40 \mathrm{ff}$.

40 Presseerklärung der Europäischen Kommission vom 17.12. 2013 Memo/13/1171.

41 Art. 30 der VO (Fn. 1), ABlEU L 158/106.

$42 \mathrm{Zu}$ den Empfehlungen und Leitlinien der ESMA für eine unionsweit einheitliche Auslegung des Kapitalmarktrechts als bloße Auslegungshilfe VeIL, aaO (Fn. 9), \$4 Rdn. $21 \mathrm{f}$; zuvor zu den delegierten Rechtsakten nach Art. 290 AEUV und den Durchführungsrechtsakten nach Art. 291 AEUV und der umstrittenen Abgrenzung derselben ebenda, $\mathbb{} 4 \mathrm{Rdn}$. $10 \mathrm{ff}$, diese können nach der Meroni-Doktrin des EuGH nicht auf die ESMA delegiert werden, $\$ 4$ Rdn. 27 Fn. 51.

43 Art. 30 Abs. 8 und 9 der VO (Fn. 1), ABlEU 158/106. 
Signal an den Markt zu geben, vielmehr kann es sich ein Prüfungsunternehmen, das laufender Aufsicht unterliegt, auf Dauer schon tatsächlich nicht leisten, sich auf Dauer gegen die Aufsicht zu stellen.

Trotz der Abmilderungen in der Endfassung und der Zurückdrängung der Rolle der ESMA zeichnet sich also über die Durchsetzung und Aufsicht doch eine Tendenz zur Verlagerung auf die europäische Ebene $a b,{ }^{44}$ wie sie, wenn dort auch deutlich weitergehend, schon derzeit im Bank- und Finanzrecht bereits vollzogen worden ist und im Kapitalmarktrecht derzeit vollzogen wird. ${ }^{45} \mathrm{Um}$ Missverständnisse zu vermeiden: Es geht an dieser Stelle nicht darum, dies abzulehnen oder zu befürworten, sondern nur darum, die Aufmerksamkeit auf einen schleichenden, in den Mitgliedstaaten wenig beachteten und unterschätzten Prozess lenken.

\section{Harmonisierung eines Kernbereichs der Abschlussprüferhaftung zwecks größerer Rechtssicherheit}

Zur Durchsetzung des vorgesehenen Pflichtenkatalogs beschränkt sich das europäische Recht beschränkt bisher - von Aufsichtsrecht abgesehen - darauf, in Art. 30 Abs. 2 der Richtlinie von 2006 den Mitgliedstaaten allgemein aufzugeben, dass diese „wirksame, verhältnismäßige und abschreckende Sanktionen" für Abschlussprüfer vorsehen müssen, die sich nicht an die Richtlinie halten. Was wirksam, verhältnismäßig und abschreckend ist, bleibt offen. Eine Studie über zivilrechtliche Haftungssysteme für die Europäische Kommission von 2001 betont die nationalen Unterschiede, eine weitere Studie hat London Economics im Auftrag der Europäischen Kommission 2005/2006 vorgelegt. ${ }^{46}$ Danach ist ein Hauptproblem der Abschlussprüferhaftung die Frage der Versicherbarkeit, insbesondere bei Großrisiken, weil die Versicherer aus diesem internationalen Markt weitgehend herausgegangen sind. ${ }^{47}$ Hier wären flexible, kumulative Lösungen von Pool- und Fremd- und Selbstversicherung zu su-

44 Die Rolle der ESMA wird, ohne dass das im Text der Verordnung angesprochen ist, über die Hintertür der Präambel doch noch dahin verstärkt, das ein Unterausschuss unter dem Vorsitz des von der ESMA benannten Mitglieds für Drittländerfragen zu bilden ist. Präambel 27 der VO (Fn. 1), ABlEU L 158/82.

45 Interessant war ein ähnlicher Versuch, die ESMA mit Eingriffsbefugnissen zu betrauen, im Bereich des Übernahmerechts, wozu es dann zwar nicht gekommen ist, aber sich eine Grauzone gebildet hat. Dazu Hopt, Europäisches Übernahmerecht, 2013, S. 108 f.

46 Dazu Ebke, aaO (Fn. 26), \$323 HGB Rdn. 245, 253 m. w. N. Neuere rechtsvergleichende Befunde bei W. Doralt, in diesem Heft, S. 273 ff unter II. 2. zu Österreich, England, Frankreich, Belgien, Italien und weiteren Ländern.

47 Dazu Van Hulle, The liability of auditors and the problem of insurability, Wallenberg Foundation Symposium, Stockholm, 30. 8.2013. 
chen. Auch würde eine Harmonisierung tief in das allgemeine Haftungsrecht der Mitgliedstaaten eingreifen, wovor sich die Europäische Kommission scheut, obschon mittlerweile durchaus Fortschritte bei der europäischen Harmonisierung auch im Kernzivilrecht zu verzeichnen sind. Die Reformvorschläge 2011 haben sich deshalb nicht auf dieses Gebiet gewagt.

Die Empfehlung der Kommission von $2008^{48}$ geht in die umgekehrte Richtung, indem sie drei Methoden zur Beschränkung der zivilrechtlichen Haftung von Abschlussprüfern und Prüfungsgesellschaften zur Wahl stellt: finanzieller Höchstbetrag, Beschränkung der Haftung auf den tatsächlichen Beitrag zum Schaden, oder Haftungsbeschränkungsvereinbarung, letztere allerdings unter gerichtlicher Kontrolle, Billigung der Aktionäre und Veröffentlichung im Anhang des Abschlusses. Die Sinnhaftigkeit dieser Richtlinie steht angesichts des engen internationalen Oligopolmarkts unter den großen Abschlussprüfungsgesellschaften und der systemischen Konsequenzen, die ein Ausscheiden eines weiteren der big four hätte, gewiss nicht in Frage. Wenn aber ein Kommissar wie McCreevey in seiner Amtszeit auf dem Gebiet des Aktien- und Wirtschaftsprüferrechts im Wesentlichen nur eine solche Schutzregelung für Wirtschaftsprüfer zustande bringt und man dann noch weiß, dass er selbst von Berufs wegen Wirtschaftsprüfer ist, dann stellt das einem solchen Amtsträger ein verheerendes Zeugnis aus.

Deutschland hat in $\$ 323$ HGB den Weg der finanziellen Haftungshöchstgrenzen gewählt. Dazu ist allerdings zu bemerken, dass die dort festgesetzten Haftungshöchstbeträge in keiner Weise sachgerecht sind. Das ist gerade auch für den Berufsstand kein Erfolg, sondern nachgerade gefährlich. Bekanntlich führt nicht selten ein zu Wenig an Reform, was zunächst nicht ganz unwillkommen sein mag, im späteren Gegenzug eher zu einem Zuviel. Aus dem Prospektrecht ist die Entwicklung bekannt, dass die auf grobe Fahrlässigkeit und mit weiteren Einschränkungen versehene sondergesetzliche Prospekthaftung der Rechtsprechung als unangemessen eng erschien mit der Folge, dass diese eine eigene, schärfere, zivilrechtliche Prospekthaftung kreierte. ${ }^{49}$ Rechtlich besser und längerfristig politisch klüger ist es deshalb, sich vernünftigen Reformen nicht zu verschließen, ja diese möglicherweise sogar anzuregen.

48 Europäische Kommission, Empfehlung zur Beschränkung der zivilrechtlichen Haftung von Abschlussprüfern und Prüfungsgesellschaften vom 5. 6. 2008, ABlEU L 162/39.

$49 \mathrm{Zu}$ beiden Prospekthaftungen M. Rотн und Kumpan, in: Baumbach/Hopt, HGB, 36. Aufl., 2013, $\$ 177$ a HGB Rdn. $59 \mathrm{ff}$ (zivilrechtliche Prospekthaftung) und (15 a) WpPG $\$ \$ 21-25$ und (15 b) VermAnlG $\$ \$ 20-22$ (sondergesetzliche Prospekthaftung). Zur Prospekthaftung des Wirtschaftsprüfers zuletzt BGH ZIP 2014, 972; zur Wirtschaftsprüferhaftung allgemeiner zuletzt BGH AG 2014, 84 und MeIXner/SchröDER, Wirtschaftsprüferhaftung, 2013; zur Haftung des Wirtschaftsprüfers für die Prüfung nach $\$ 53$ HGrG Kersting, ZIP 2014, 2420. 
Eine europäische Harmonisierung des Kernbereichs der Abschlussprüferhaftung erscheint aus diesen Gründen und aus solchen der Rechtssicherheit für alle Beteiligten - Abschlussprüfer, Gesellschaften, Aktionäre und geschädigten Dritte, aber auch für den Berufsstand ingesamt - sinnvoll und weiterführend. ${ }^{50}$ Dabei sollten Mindestvorgaben bezüglich der Ausgestaltung der zivilrechtlichen Haftung des gesetzlichen Abschlussprüfers nicht nur gegenüber der zu prüfenden Gesellschaft und möglicherweise ihren Aktionären, sondern auch gegenüber Dritten ${ }^{51}$ - darin liegt die wirklich Brisanz - gemacht werden, dies zumal als die Mitgliedstaaten insoweit stark differieren mit England und im Ergebnis Deutschland auf der einen Seite und Frankreich, Belgien, Italien, Schweiz und Slovenien auf der anderen Seite der Skala, wenn dort auch ganz überwiegend mit Begrenzungen ${ }^{52}$. Dafür plädiert im Ergebnis auch W. Doralt, ${ }^{53}$ in grundsätzlicher Übereinstimmung mit anderen. Es ist keine Frage, dass nicht erst die Einigung auf diese Mindeststandards schwierig sein wird, sondern bereits in der Sache erhebliche Abgrenzungs- und Inhaltsfragen auftreten. Man denke nur an die umfangreiche deutsche Rechtsprechung zur Prospekthaftung der Wirtschaftsprüfer. Diesen Schwierigkeiten und anfänglichen Unsicherheiten könnte mit einer Empfehlung der Europäischen Kommission Rechnung getragen werden. Im Übrigen ist Rom auch nicht an einem Tag erbaut worden.

\section{Internationalisierung des Wirtschaftsprüferrechts}

Über die Frage, wieweit die europäische Rechtsangleichung gehen soll, wird bekanntlich nicht nur auf dem Gebiet der Abschlussprüfung gestritten, sondern allgemeiner zum europäischen Gesellschaftsrecht und darüber hinaus. Das ist ein weites Feld, das schon in der Würdigung des Aktionsplans der Kommission von 2012 angesprochen wurde,${ }^{54}$ hier aber nicht betreten werden soll. In einem weiteren Teil des Symposiums ging es spezieller um die Internationalisierung der Abschlussprüfung. Das ist etwas ganz anderes, wie Köhler ${ }^{55}$ und Merkt $^{56}$ ausführen. Harmonisierung kommt von oben, also durch Regu-

50 Zum Einwand des Wettbewerbs der Gesetzgeber (im europäischen Gesellschaftsrecht im Zusammenhang mit dem Aktionsplan der Europäischen Kommission von 2012) Hopt, ZGR 2013, 165, $181 \mathrm{ff}$.

51 Zur Dritthaftung des Abschlussprüfers kapitalmarktorientierter Unternehmen SEIBT/ Wollenschläger, Der Betrieb 2011, 1378; kritisch zur bejahenden Rechtsprechung des öOGH Zehetner, ÖZW 2013, 78, aber W. Doralt, in diesem Heft, S. 289 unter III. 3. b) aa): in der Lehre mittlerweile akzeptiert, m. w. N.

52 Dazu W. Doralt, in diesem Heft, S.285 ff unter III. 3.

53 W. Doralt, in diesem Heft, S. $291 \mathrm{f}$ unter III. 4.

54 Ausführlich Норт, ZGR 2013, 165.

55 Zur Arbeit des IAASB KöHLER, in diesem Heft, S. $204 \mathrm{ff}$.

56 Zur Bedeutung der ISA für die Abschlussprüfung in Europa und Deutschland MerkT, in diesem Heft, S. $215 \mathrm{ff}$. 
lierer aus Brüssel und Strassburg und über den Europäischen Rat aus den Mitgliedstaaten selbst. Bei der Internationalisierung geht es demgegenüber um eine freiwillige Koordination der Berufsträger selbst, die es für sinnvoll, wenn nicht geboten ansehen, auf internationale, ja globale Märkte und deren Erwartungen zu reagieren. So ist auch die Arbeit des IAASB, des International Auditing and Assurance Standard Board, zu verstehen. Diese Harmonisierung von unten beruht auf dem Erfahrungsschatz der Betroffenen und ist selfregulation im besten Sinne des Wortes. Das heißt gewiss nicht, dass das $\mathrm{Zu}$ standekommen dieser self-regulation leicht wäre. Die nationalen rechtlichen und kulturellen Unterschiede - man spricht von Pfadabhängigkeit (path dependence) - sind riesig, und ohne Kompromisse geht es auch dort nicht ab. Gerade deswegen ist es wichtig, dass sich Deutschland an diesen Arbeiten beteiligt und seinen fairen Anteil an der Kostentragung übernimmt. Nicht zuletzt angesichts einer gewissen Dominanz der angelsächsischen Teilnehmer ist die Zurückhaltung, um nicht zu sagen Verweigerung, höchst kurzsichtig, die die Bundesrepublik diesbezüglich geübt hat. Nach der Abschlussprüferrichtlinie sind die Mitgliedstaaten verpflichtet, für die Anwendung der internationalen Prüfungsstandards, insbesondere der International Standards on Auditing (ISA), zu sorgen, vorbehaltlich nur des Anerkennungsverfahrens der Europäischen Kommission. ${ }^{57}$ Das ist im Vergleich zu einer unmittelbaren Rechtssetzung durch Verordnung oder mittelbar durch eine Richtlinie der eindeutig bessere Weg.

Wenn es richtig ist, dass der IAASB auf die Erwartungen des Wirtschaftsprüfungsmarktes international und in der Europäischen Union reagiert, dann ist es wichtig, mehr über die Funktionsbedingungen des Wirtschaftsprüfungsmarktes zu erfahren, wie näher von Poll behandelt. ${ }^{58}$ Dazu hat nach Presseberichten $^{59}$ jüngst der Vorstandssprecher des Instituts der Wirtschaftsprüfer Naumann geäußert, die Wirtschaftsprüfung stehe „vor großen Veränderungen. Die klassisch, gesetzlich vorgeschriebene Prüfung wird an Bedeutung verlieren, sie wird zur reinen Ordnungsmäßigkeitsprüfung. " Die Wirtschaftsprüfung müsse künftig vielmehr beantworten, wo die Risiken im Geschäftsmodell des Mandanten liegen. Das impliziert diffizilste Fragen, wie es im engen Oligopol der Big Four, der darunter liegenden mittelgroßen Prüfungsgesellschaften und der zahlreichen kleinen aussieht, wer von diesen sich mit Aussicht auf Erfolg auf dem weiteren europäischen Markt tummeln kann und wie die geplanten europäischen Regelungen, wie immer sie letztlich aussehen werden, sich auf diesen Wettbewerb inhaltlich, kostenmäßig und damit marktlich auswirken werden. ${ }^{60}$

57 Art. 26 der Richtlinie (Fn. 2), ABlEU L 158/211. Zu den Schwierigkeiten dabei eingehend Merkt, in diesem Heft, S. $222 \mathrm{ff}$ unter III.-V.

58 Poll, in diesem Heft, S. $234 \mathrm{ff}$.

59 Die Wirtschaftsprüfung wird zur Risikoprüfung, FAZ Nr. 185, 12. 8. 2013, S. 21.

$60 \mathrm{Vgl}$. ansatzweise empirisch zu den möglichen Auswirkungen der EU-Regulierungsent- 


\section{Abschlussprüfer zwischen Vorstand und Aufsichtsrat}

Abschlussprüfung und Abschlussprüfer sind, was mittlerweile Allgemeingut ist, ein wichtiger Teil der Corporate Governance. ${ }^{61}$ Die Abschlussprüfer haben die Rolle eines „public watchdog“ an den Kapitalmärkten. ${ }^{62}$ Die vereinzelt zu hörende Aussage, die Abschlussprüfung gehöre nicht in den Kontext der Corporate Governance ist schon deshalb unverständlich, weil der Deutsche Corporate Governance Kodex Rechnungslegung und Abschlussprüfung ausführlich einbezieht. ${ }^{63}$ Für die funktionale Zusammengehörigkeit ist bezeichnend, dass der Altmeister des englischen Aktienrechts in seinem großen Lehrbuch „Principles of Modern Company Law“ einen langen eigenen Teil über „Account and Audit" aufgenommen hat. ${ }^{64}$ Als Teil der Corporate Governance ist die Regulierung der Abschlussprüfer in Deutschland und Europa durch die Erfahrungen mit Enron und dem US-amerikanischen Sarbanes-Oxley Act vom 30. Juli 2002 (nebst Folgeregelungen der SEC und der NYSE) beeinflusst, wenn auch nicht geprägt worden. Letzterer Act geht weit in den Unabhängigkeitsanforderungen und der (bloß internen) Trennung von Prüfung und Beratung (dazu die Liste in Section 201). Die Wissenschaft unterscheidet bekanntlich zwischen interner Corporate Governance, also Kontrolle durch die Aktionäre bzw., soweit diese damit überfordert sind, durch entsprechende aktienrechtliche Rahmenbedingungen, und der externen Corporate Governance, im wesentlichen einer solchen durch die Märkte, allen voran durch den Markt für Unternehmenskontrolle (market for corporate control). ${ }^{65} \mathrm{Die} \mathrm{Ab}-$ schlussprüfung steht zwischen interner und externer Corporate Governance. Der Abschlussprüfer wird anders als früher nicht mehr als Organ der Gesellschaft angesehen. Er ist aber trotz seiner Unabhängigkeit auch nicht ein völlig

würfe zur Abschlussprüfung auf deutsche Unternehmen, ERFKAMP/Janke, WPg 2013, 264.

61 Vgl. M. Müller, Der Prüfungsbericht des Abschlussprüfers als Instrument der Corporate Governance, 2013; Hommelhoff, Die EU-Reform der Abschlussprüfung vor dem Hintergrund der deutschen Corporate Governance, Gegliederte Thesen, November 2011; MatTheus, Die Rolle des Abschlussprüfers in der Corporate Governance, in: Hommelhoff/Hopt/v. Werder, Handbuch Corporate Governance, 2. Aufl., 2009, S. 563; auch Hopt/Merkт, aaO (Fn. 3), Überblick vor $\$ 316$ HGB Rdn. 6 f.

62 Arbeitskreis Bilanzrecht Hochschullehrer Rechtswissenschaft, NZG 2012, 294, 295.

63 Deutscher Corporate Governance Kodex, Fassung vom 13.5.2013, Abschnitt 7; dazu Ringleb und Kremer, in: Ringleb/Kremer/Lutter/v. Werder, Deutscher Corporate Governance Kodex, Kommentar, 5. Aufl., 2014, Rdn. 1173-1248.

64 Davies/Worthington, Gower \& Davies: Principles of Modern Company Law, 9th ed., London 2012, Part Five Account and Audit, p. 751-857. Zu den möglichen Regulierungsstrategien ebenda, p. 808 et seq.

65 Zum market for corporate control Hopt, aaO (Fn.45), S.11f, 84 ff. Auch Ders., Takeover Defenses in Europe: A Comparative, Theoretical and Policy Analysis, Columbia Journal of European Law 20.2 (2014) 249 at 261-269. 
außenstehender Kontrolleur, wie etwa die Wirtschaftsprüferkammer oder die BaFin. In der internationalen Fachsprache ist der Abschlussprüfer vielmehr ein gatekeeper, ${ }^{66}$ also jemand, der für Ordnung in dem Etablissement sorgt. Der Japankenner wird hier an die beiden grimmigen Wächter denken, die links und rechts am Eingang buddhistischer Tempel stehen. So grimmig darf man sich die Abschlussprüfer natürlich nicht vorstehen. Es geht - um im Bild zu bleiben - eher umgekehrt darum, dafür zu sorgen, dass diese nicht von vornherein lächelnd einladen, sondern ihre gatekeeping function auch wirklich erfüllen.

Um dieser Gatekeeping-Funktion zwischen innen und außen gerecht zu werden, kommt es entscheidend darauf an, die Aktiengesellschaft nicht nur von außen zu prüfen, sondern von innen zu kennen. Der Informationsfluss in der Gesellschaft vom Vorstand zum Aufsichtsrat und von diesen beiden zum Abschlussprüfer, die Zusammenarbeit zwischen Aufsichtsrat und Abschlussprüfer ${ }^{67}$ und, mit beidem verbunden, die zentral wichtige Gesprächskultur zwischen diesen dreien sind für die Unternehmenskontrolle schlechthin essentiell. Zentrum dieses Geschehens ist jedenfalls in großen, börsennotierten Aktiengesellschaften der Prüfungsausschuss, der ja ein gewisses Vorbild in den audit committees angelsächsischer Prägung ${ }^{68}$ hat und im Deutschen Corporate Governance Kodex und zuletzt auch durch den Gesetzgeber mehrfach angesprochen und in Pflicht genommen worden ist. ${ }^{69}$ Die Abschlussprüferrichtlinie enthält strengere Regelungen über den Prüfungsausschuss, und die Abschlusssprüferverordnung nimmt den Prüfungsausschuss vor allem bei der Bestellung der Abschlussprüfer in die Pflicht. ${ }^{70}$ Wichtig wäre es deshalb, mehr aus der Praxis des Prüfungsausschusses in der börsennotierten AG zu erfah-

66 Dazu Kraakman/Armour/Davies/Enriques/Hansmann/Hertig/Hopt/Kanda/ Rock, The Anatomy of Corporate Law, A Comparative and Functional Approach, 2 d ed., Oxford 2009, p. 128 et seq.

67 IDW Positionspapier zur Zusammenarbeit zwischen Aufsichtsrat und Abschlussprüfer, IDW Fachnachrichten 6/2012, 339; auch SchüpPEN, ZIP 2012, 1317; empirische Befunde bei Velte, AG 2009, 102.

68 Zur Rolle des audit committee des board für die Corporate Governance Davies/ Worthington, aaO (Fn. 64), p. 825 et seq.

69 Art. 100 Abs. 5, 107 Abs. 3 Satz 2, Abs. 4, 124 Abs. 3 Satz 2, 171 Abs. 1 Sätze 2 und 3 u. a. AktG; $\$ 324$ HGB Prüfungsausschuss, dazu einführend Норт/Меrкт, aаO (Fn.3), \324 HGB, Literaturnachweise ebenda Rdn. 1. Zur Compliance aus der Sicht des Prüfungsausschusses Editorial Hommelhoff, WPg 22/2013, I.

70 Art. 39 der Richtlinie (Fn. 2), ABlEU L 158/220; Art. 16 der VO (Fn. 1), ABlEU L 158/96, insbesondere Empfehlung an den Aufsichtsrat für die Bestellung von Abschlussprüfern im Anschluss an ein formalisiertes Auswahlverfahren. Die Stärkung des Prüfungsausschusses begrüßt Arbeitskreis Bilanzrecht Hochschullehrer Rechtswissenschaft, NZG 2012, 294, 295; auch Lanfermann/Maul, BB 2012, 627; Schüppen, ZIP 2012, 1317, 1319f; zur Rolle der Prüfungsausschüsse auch die Beiträge in Audit Committee Quarterly, Sonderpublikation für M. D. Kley, Prüfungsausschüsse: die Alleskönner?, Nov. 2014. 
ren. Es wäre interessant zu wissen, ob es gelungen ist oder voraussichtlich noch gelingen wird, den Abschlussprüfer zu einem echten sparring partner des Aufsichtsrats zu machen. Bedenkenswert ist auch der aus der Wissenschaft gemachte Vorschlag, zu verlangen, dass bei kapitalmarktorientierten Kapitalgesellschaften ( $\$ 264 \mathrm{~d}$ HGB) im Aufsichtsrat bzw. Prüfungsausschuss Sachverstand auf beiden Gebieten Rechnungslegung und (nicht: oder) Abschlussprüfung vorhanden sein muss. ${ }^{71}$

71 Vgl. bisher $\$ \int 100$ Abs. 5, 107 Abs. 4 AktG. 